Société d'histoire de la révolution de 1848 et des

révolutions du XIXe siècle

$47 \mid 2013$

Quel est l'avenir du XIX $X^{\mathrm{e}}$ siècle ?

\title{
Laura FOURNIER-FINOCCHIARO et Tanja-Isabel HABICHT [dir.], Gallomanie et gallophobie. Le mythe français en Europe au XIX ${ }^{e}$ siècle
}

Rennes, Presses universitaires de Rennes, 2012, 315 p. ISBN :

978-2-7535-2039-4. 18 euros.

Pierre Ranger

\section{(2) OpenEdition}

Journals

Édition électronique

URL : http://journals.openedition.org/rh19/4624

DOI : $10.4000 /$ rh 19.4624

ISSN : $1777-5329$

Éditeur

La Société de 1848

\section{Édition imprimée}

Date de publication : 31 décembre 2013

Pagination : 229-230

ISSN : 1265-1354

\section{Référence électronique}

Pierre Ranger, «Laura FOURNIER-FINOCCHIARO et Tanja-Isabel HABICHT [dir.], Gallomanie et gallophobie. Le mythe français en Europe au XIXe siècle », Revue d'histoire du XIXe siècle [En ligne], 47 | 2013, mis en ligne le 28 janvier 2014, consulté le 22 septembre 2020. URL : http:// journals.openedition.org/rh19/4624; DOl : https://doi.org/10.4000/rh19.4624 


\title{
Laura FOURNIER-FINOCCHIARO et Tanja-Isabel HABICHT [dir.], Gallomanie et gallophobie. Le mythe français en Europe au XIX ${ }^{e}$ siècle
}

\author{
Rennes, Presses universitaires de Rennes, 2012, 315 p. ISBN :
}

978-2-7535-2039-4. 18 euros.

Pierre Ranger

\section{RÉFÉRENCE}

Laura FOURNIER-FINOCCHIARO et Tanja-Isabel HABICHT [dir.], Gallomanie et gallophobie. Le mythe français en Europe au XIX ${ }^{e}$ siècle, Rennes, Presses universitaires de Rennes, 2012, 315 p. ISBN : 978-2-7535-2039-4. 18 euros.

1 L'ouvrage est le résultat d'un colloque tenu en 2011 à l'université de Caen pour « confronter les multiples représentations de la France à l'étranger» (p. 8) et analyser le rôle du «mythe français » au sein des processus de construction nationale en Europe au XIXe siècle. L'introduction précise que la France « impose à tous les pays européens une confrontation» avec son modèle, chaque nation se définissant "au prisme de l'autre» (p. 9). Ce travail s'inscrit dans la continuité des écrits d'Anne-Marie Thiesse et de Benedict Anderson, l'imprimé constituant une bonne part du matériel mobilisé par les auteurs. Il confirme l'intérêt actuel des historiens du nationalisme pour les études transnationales. Le «mythe français» est un bel outil d'analyse pour démontrer la pertinence de cette nouvelle approche.

2 L'absence d'une définition initiale de l'objet réduit cependant la cohérence d'ensemble $\mathrm{du}$ projet, invitant certains contributeurs à limiter leur étude aux relations politiques et culturelles. C'est trop peu lorsqu'un mythe et sa survivance au sein des imaginaires nationaux sont soumis à l'analyse. Beaucoup de chapitres sont d'ailleurs allés au-delà 
de cette approche. Le «mythe français » y est perçu comme un outil utilisé pour tracer les contours d'une culture nationale donnée en intériorisant la différence. Il ne s'agit pas de penser une relation à l'autre mais de se définir en nation. C'est donc au fil de la lecture que le «mythe français » prend forme, au gré de représentations répétées au sein des différents espaces étudiés. Paris, capitale des révolutions et moderne Babylone, se trouve ainsi dépeinte à plusieurs reprises, entre fascination et rejet. Ces perceptions sont aussi celles des groupes socio-politiques concernés, issus de l'élite lettrée libérale, parfois républicaine.

3 Au regard du nombre important de contributions réunies (dix-huit en tout), je présenterai les idées fortes contenues dans les trois parties qui composent l'ouvrage ainsi que quelques articles.

4 Le premier thème confronte l'attitude de diverses nations envers la France. Le « mythe français » y apparaît relativement protéiforme même si sa composante hégémonique, voire menaçante lorsqu'elle est révolutionnaire, est récurrente. Au moins pour la première moitié du XIX siècle, s'affirmer face à la présence politique et culturelle française semble un passage obligé pour beaucoup de nationalistes. Ainsi, Laura Fournier-Finocchiaro analyse les relations de Mazzini à la France, donnant au personnage le statut d'un promoteur de l'émancipation nationale en Italie et en Europe plutôt que d'un gallophobe. Selon Tom Verschaffel, l'image de la France est autant nécessaire aux promoteurs de la nation belge dont l'histoire se construit au travers de la notion de domination étrangère. La France est au cœur de ce récit. Certains nationalistes belges la décrivent comme un danger pour la pérennité territoriale et culturelle de la jeune nation. Un autre pan du «mythe français», cette fois monarchique, est présenté par Tanja-Isabel Habicht qui analyse les emprunts que Louis II de Bavière fait à la France de Louis XIV pour montrer son opposition au processus d'unification allemande.

5 Le deuxième thème est consacré aux parcours d'écrivains et d'artistes qui, entre gallophobies et gallomanies, ont utilisé la France comme sujet moteur de leur œuvre. Ils ont ainsi enrichi et porté le «mythe français» en Europe. Ces contributions, qui insistent sur l'essence créatrice du mythe, en expliquent aussi les ressorts idéologiques, comme le montrent bien deux articles portant sur les gallophobes italiens. Christian Del Vento analyse la gallophobie de Vittorio Alfieri et nous fait découvrir un écrivain aux idées radicales mais profondément déçu par la tournure des événements postrévolutionnaires français. Stéphanie Lanfranchi étudie trois auteurs (Foscolo, Leopardi et Alfieri de nouveau), dont la gallophobie se lit comme une déclaration d'intention culturelle et politique en faveur de l'italianité contre l'omniprésence française. Les deux chapitres se font écho lorsqu'ils expliquent la réinterprétation du mythe gallophobe par les nationalistes italiens de la fin du siècle comme la justification à leurs revendications identitaires hégémoniques, démontrant combien les mythes nationaux se nourrissent les uns des autres.

6 La troisième partie propose des outils d'analyse sans lesquels on ne peut comprendre la résilience de certaines représentations de la France en Europe. Les contributeurs y analysent avec clarté les outils de diffusion du mythe au sein des populations européennes, des élites lettrées aux écoliers. On y découvre un «mythe français » élément d'éducation et outil didactique. Iris de Barros-Sousa étudie les dictionnaires de traduction franco-portugais qui ouvrent deux lexicographies nationales l'une à l'autre. La technique lexicographique française participe ainsi à la codification lexicale du 
portugais à la toute fin du XVIII ${ }^{e}$ siècle. Laurence Boudart avance dans le siècle et s'intéresse à une autre frange de la population en étudiant les manuels scolaires belges. La France, sans être un sujet central, offre matière à comparaison pour mieux exalter les atouts de la jeune nation. En guise de conclusion générale, Jean-Claude Lescure propose une réflexion sur le déclin linguistique français au tournant des $\mathrm{XIX}^{\mathrm{e}}$ et $\mathrm{XX}^{\mathrm{e}}$ siècles. Dans un contexte de compétition entre langues européennes, la France travaille à son mythe par le développement des réseaux de la francophonie.

7 Cette contribution collective à l'histoire de la construction des nations est utile. Elle rappelle que la formation des critères nationaux au XIX ${ }^{\mathrm{e}}$ siècle tient pour beaucoup aux représentations que l'on se fait de l'autre. La France occupe dans ce processus une place centrale par sa surface politique et culturelle. Néanmoins, ce phénomène ne pouvant être réduit à son seul cas, cet ouvrage appelle une réflexion plus globale sur l'ensemble des mythes transnationaux européens. 